\title{
Accurate antenna reflector loss measurements for radiometer calibration budget
}

\section{Skou, Niels}

Published in:

Geoscience and Remote Sensing Symposium, 1996. IGARSS '96. 'Remote Sensing for a Sustainable Future.', International

Link to article, DOI:

10.1109/IGARSS.1996.516463

Publication date:

1996

Document Version

Publisher's PDF, also known as Version of record

Link back to DTU Orbit

Citation $(A P A)$ :

Skou, N. (1996). Accurate antenna reflector loss measurements for radiometer calibration budget. In Geoscience and Remote Sensing Symposium, 1996. IGARSS '96. 'Remote Sensing for a Sustainable Future.', International (pp. 749-753). IEEE. https://doi.org/10.1109/IGARSS.1996.516463

\section{General rights}

Copyright and moral rights for the publications made accessible in the public portal are retained by the authors and/or other copyright owners and it is a condition of accessing publications that users recognise and abide by the legal requirements associated with these rights.

- Users may download and print one copy of any publication from the public portal for the purpose of private study or research.

- You may not further distribute the material or use it for any profit-making activity or commercial gain

- You may freely distribute the URL identifying the publication in the public portal 


\title{
Accurate Antenna Reflector Loss Measurements for Radiometer Calibration Budget
}

\author{
Niels Skou \\ Danish Center for Remote Sensing, Electromagnetics Institute \\ Technical University of Denmark (TUD), B-348, DK-2800 Lyngby, Denmark. \\ Phone: (45) 458814 44, Fax: (45) 4593 16 34, E-mail: ns@emi.dtu.dk
}

\begin{abstract}
Antenna reflector losses may play an important role in the calibration budget for a microwave radiometer. If the losses are small they are difficult to measure by traditional means. However, they can be assessed directly by radiometric means using the sky brightness temperature as incident radiation. The paper describes how such measurements are carried out as well as a suitable experimental set-up. The main reflector of the European Space Agency's MIMR system is used to demonstrate the principle.
\end{abstract}

\section{INTRODUCTION}

The fundamental pre-launch calibration of spaceborne radiometer systems is normally carried out with the instrument inside a thermal vacuum chamber which also includes a calibration target viewed by the radiometers antenna system. The calibration target is very accurately temperature controlled and is able to provide brightness temperatures over a wide range, typically some $77 \mathrm{~K}$ to some $300 \mathrm{~K}$. Such a target is difficult and expensive to develop - especially if it has to be large in order to serve a large aperture system. A thermal vacuum chamber is also not a cheap and trivial installation - especially if it has to be large. For these reasons it may be necessary to dismount the antenna reflector and carry out the calibration with the feed horns viewing the calibration target. Thus reflector losses must be accurately assessed so that they can be compensated for in the data analysis.

The reflection coefficient for a specimen of the reflector material can be measured on a network analyzer. However, if the reflection coefficient is close to 1 , which it will be for a decent reflector, it turns out to be difficult if not impossible to achieve the necessary measurement accuracy. On the other hand it is really the emissivity of the reflector material we need for the radiometric calibration correction, and this can be measured directly and accurately down to very small values by radiometric means.

\section{MEASUREMENT SETUP}

The reflector to be measured, or a specimen of its material, is placed in a large metal bucket together with a radiometer and a suitable horn antenna. The device under test reflects the clear sky brightness temperature into the radiometer horn. The bucket diverts antenna sidelobes and spillover towards clear sky.

Figure 1 shows how the reflector emissivity is measured in a 3-step process using the sky temperature as radiation 0-7803-3068-4/96\$5.00@1996 IEEE

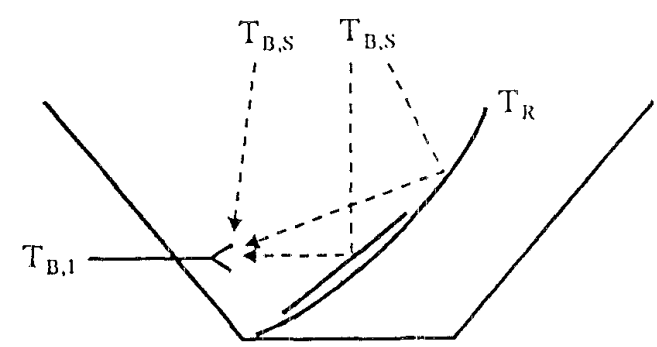

(a) Reflector and lossiess plate

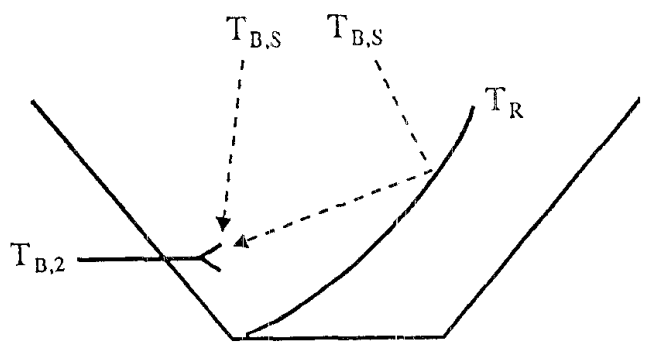

(b) Reflector

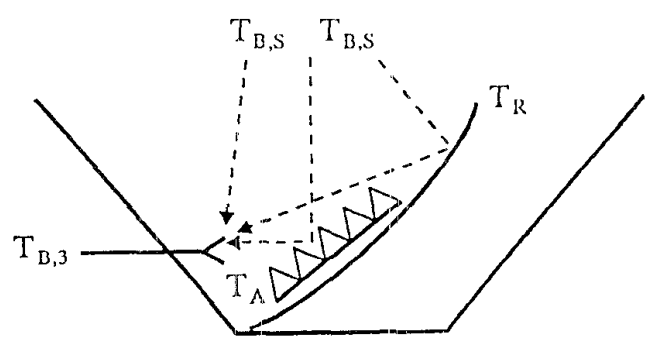

(c) Reflector and absorber

Figure 1: Measurement schedule

source. First the sky temperature $\mathrm{T}_{\mathrm{B}} \mathrm{S}$ is measured using a lossless plate (in this case a silver plated aluminium plate):

$\mathrm{T}_{\mathrm{B}, 1}=\mathrm{n} \cdot \mathrm{T}_{\mathrm{B}, \mathrm{S}}+(1-\mathrm{n}) \mathrm{T}_{\mathrm{B}, \mathrm{S}}=\mathrm{T}_{\mathrm{B}, \mathrm{S}}$ 
where $n$ is the beam filling factor of the plate. $n$ is assumed large and the emissivities of the reflector and the bucket are assumed small. This step can be regarded as an inter-calibration of the radiometer and the sky temperature. Secondly, the reflector alone is measured:

$$
\mathrm{T}_{\mathrm{B}, 2}=\mathrm{n}\left(\mathrm{T}_{\mathrm{R}} \cdot \varepsilon+\mathrm{T}_{\mathrm{B}, \mathrm{S}}(1-\varepsilon)\right)+(1-\mathrm{n}) \mathrm{T}_{\mathrm{B}, \mathrm{S}}
$$

where $T R$ is the temperature of the reflector and $\varepsilon$ is its emissivity. If the reflector is very good (low loss) the difference between $T_{B}, 1$ and $T_{B}, 2$ is small. Finally, in the 3rd step an absorber having the same physical size as the lossless plate is measured:

$$
\mathrm{T}_{\mathrm{B}, 3}=\mathrm{n} \cdot \mathrm{T}_{\mathrm{B}, \mathrm{A}}+(1-\mathrm{n}) \cdot \mathrm{T}_{\mathrm{B}, \mathrm{S}}
$$

where $T_{B, A}$ is the physical (hence also the brightness) temperature of the absorber.

Solving the above 3 equations we find:

$$
\begin{aligned}
& n=\frac{T_{B, 3}-T_{B, 1}}{T_{B, A}-T_{B, 1}} \\
& \varepsilon=\frac{T_{B, 2}-T_{B, 1}}{n\left(T_{R}-T_{B, 1}\right)}
\end{aligned}
$$

It is seen that $\mathrm{T}_{\mathrm{B}, \mathrm{S}}$ has to be constant throughout the experiment. So, only clear days without clouds can be used. Also the radiometer must be stable throughout an experiment. If the reflector is very good the difference $T_{B}, 2-T_{B}, 1$ (which is the dominating factor in determining the emissivity) become small and the requirement to stability become stringent. The crucial steps (a) and (b) are measured several times alternating between them. The time between successive measurements is kept to 2 minutes. Thus the time difference (important versus stability) is short and measurements of the same situation is repeated enabling drifts to be checked.

The aluminium lined bucket bas an opening of $3 \times 3 \mathrm{~m}$ and a height of $1.2 \mathrm{~m}$. The lossless plate is $0.5 \times 0.5 \mathrm{~m} .3$ noise-injection radiometers $(5,17$ and $34 \mathrm{GHz})$ with associated $25 \mathrm{~dB}$ standard gain horns are used for the measurements. The brighmess temperatures are measured with a standard deviation of $0.1 \mathrm{~K}$.

The $5 \mathrm{GHz}$ standard gain horn is very large compared with the lossless plate and it has been found useful to also employ a smaller horn (see later).

\section{THEORETICAL EMISSIVITY}

With the notation $\mathrm{R}_{\mathrm{S}}=$ surface resistivity, and $\eta=$ impedance of intrinsic medium, the power reflectivity for a good conductor is:

$$
\rho=1-\frac{4 R_{s}}{\eta}
$$

$\eta=377$ when the intrinsic medium is air as in our case. $\rho=1-\varepsilon$ in case of no transmission which we can assume with good confidence, considering our relatively thick metal plates.

For the surface resistivity we find: $R_{S}=\sqrt{\frac{\pi f \mu}{\sigma}}$ or for non-magnetic material:

$$
R_{\mathrm{s}}=\sqrt{\frac{\pi \cdot f \cdot \mu_{0}}{\sigma}}=2 \pi \cdot \sqrt{\frac{f}{\sigma \cdot 10^{7}}}
$$

where $\sigma$ is the conductivity, and $f$ the frequency. Hence we find

$$
\varepsilon=\frac{1}{15} \sqrt{\frac{f}{\sigma \cdot 10^{7}}}
$$

Inserting the conductivity for typical metals we find the values shown in Table 1.

\begin{tabular}{|l|c|c|c|}
\hline & Conductivity & \multicolumn{2}{|c|}{ Emissivity } \\
& $\mathrm{s}(\mathrm{s} / \mathrm{m})$ & $17 \mathrm{GHz}$ & $34 \mathrm{GHz}$ \\
\hline Silver & $6.17 \times 10^{7}$ & 0.00035 & 0.00049 \\
Copper & $5.80 \times 10^{7}$ & 0.00036 & 0.00051 \\
Aluminium & $3.72 \times 10^{7}$ & 0.00045 & 0.00064 \\
Brass & $1.57 \times 10^{7}$ & 0.00069 & 0.00098 \\
Iron & $1.12 \times 10^{7}$ & $0.00082 *$ & $0.0012 *$ \\
\hline
\end{tabular}

* neglecting magnetic properties at frequency in question

Table 1: Emissivity of some conductors

It is seen that the difference between silver and copper is minute but also aluminium is very close.

If we want to measure emissivities in the 0.01 range then the silver plate can be considered lossless. However, if emissivity measurements in the range around 0.001 is wanted then corrections are needed.

\section{MEASUREMENTS ON METAL PLATES}

From preliminary network analyzer measurements it is expected that the emissivity of the MIMR reflector is quite small. Hence, it is of interest to assess "emissivity resolution" i.e. the lower limit of what can be measured with the present setup. To this end different metal plates have been measured against the "lossless" plate in the bucket. Like for the normal measurement procedure the test plate and the lossless plate are measured several times alternating between them and with 2 minutes between measurements. Also the absorber is measured to determine the beam filling factor the usual way. 
The test plates are of course exactly the same size as the "lossless" plate.

Figure 2 presents results from a preparatory exercise measuring the difference between an iron plate and the lossless plate. The magnetic properties of the iron plate is not known so the actual value of the emissivity is not known. It is evident from looking at the bar diagram in Figure 2 that the iron plate has an emissivity large enough to be measurable by the method employed. The reflected brightness temperatures are in the iron case systematically larger than in the lossless case. By averaging we find for iron: $\mathrm{T}_{\mathrm{B}, 2}=17.94 \mathrm{~K}$, for the lossless case: $\mathrm{T}_{\mathrm{B}, 1}=17.50 \mathrm{~K}$. Inserting in formulas (1) and (2), we find: $n=0.9814$ and $\varepsilon=0.0017$. The beam filling factor is good. The emissivity value and the systematic behaviour of the bar diagram lead us to state that the method can measure emissivity values somewhat smaller than 0.0017 .

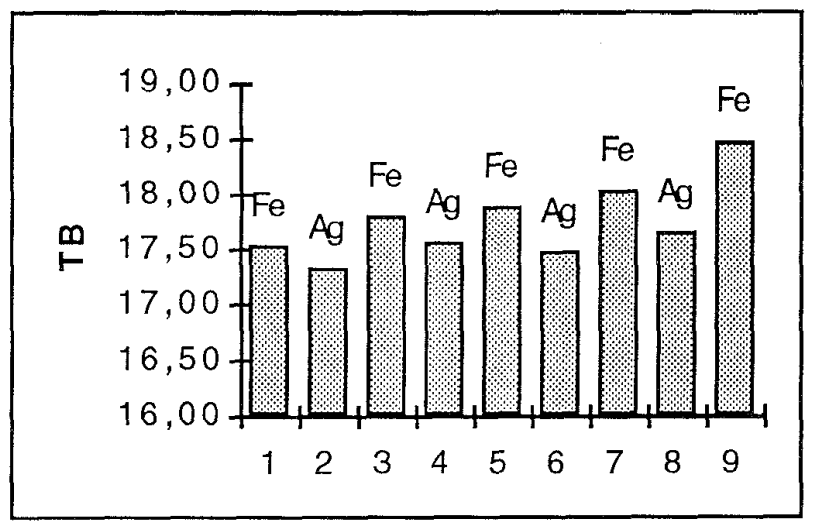

Figure 2: 34 Ghz measurement of iron and silver plates

In an attempt to find the limiting value of emissivity that we can measure, a brass plate is used in the following experiments. Figure 3 reports a $17 \mathrm{GHz}$ measurement and it is noted that good stability prevails but there is no systematic pattern in the bar chart. The calculated emissivity is as low as $\varepsilon=-0.0002$ also indicating that we cannot see the difference between brass and silver. Hence, we cannot measure the emissivity of brass at $17 \mathrm{GHz}(0.00069$ theoretically).

The next experiment, see Figure 4, concerns the measurement of brass at $34 \mathrm{GHz}$. At this frequency the theoretical emissivity is $\varepsilon=0.001$. A careful inspection of the bar chart shows that in all cases, but measurement "4", going from brass to silver results in lower brightness temperature while going from silver to brass results in higher brightness temperature. Hence, there is a systematic and reasonable pattern in the bars and we can feel confident that we can perform an emissivity assessment. By proper averaging and using the usual formula we find $\varepsilon=0.0008$ which is in fair agreement with the theoretical value bearing in mind that it is not corrected for losses in the so-called "lossless" plate.

Hence, it is seen that we can measure an emissivity of 0.001 . However, the results from similar other experiments not reported here show that in some cases we can see the difference between the lossless plate and the brass plate, in others not. In no case can a difference be detected at $17 \mathrm{GHz}$.

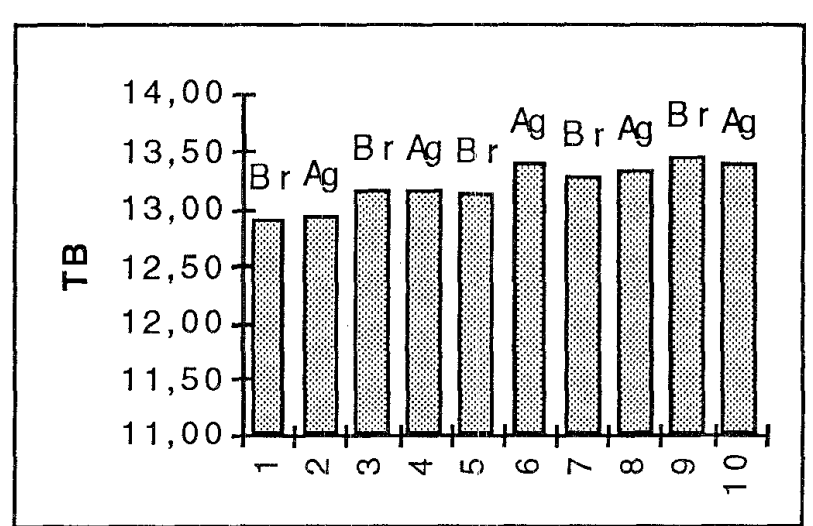

Figure 3: $17 \mathrm{GHz}$ measurement of brass and silver plates

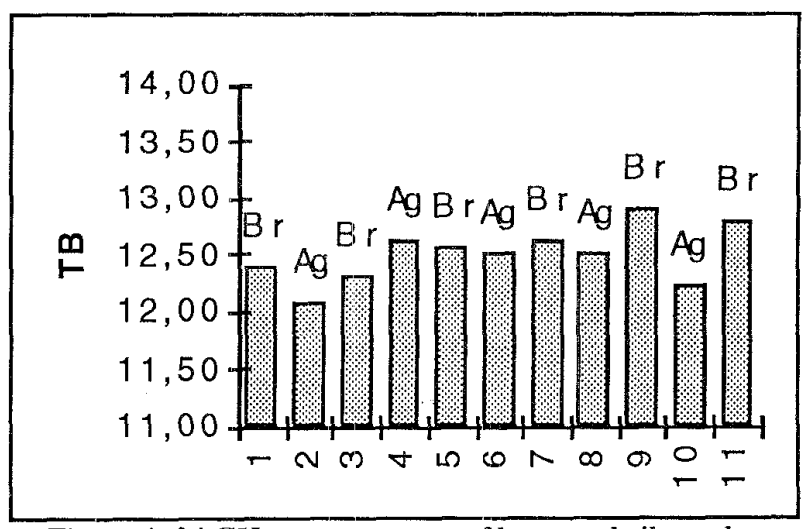

Figure 4: $34 \mathrm{GHz}$ measurement of brass and silver plates

This indicates that even this figure is marginal and we can certainly not measure smaller values. We conclude that the lower limit for emissivity measurable by the present setup is 0.001 .

\section{MIMR REFLECTOR MEASUREMENTS}

In the following a few examples of measurements on the MIMR reflector itself shall be presented. Figure 5 shows 34 $\mathrm{GHz}$ results. (a) and (b) refer to the measurement steps, see Figure 1. Stability is good and by proper averaging we find:

$\mathrm{T}_{\mathrm{B}, 1}=13.02 \mathrm{~K}, \mathrm{~T}_{\mathrm{B}, 2}=13.07 \mathrm{~K}, \mathrm{~T}_{\mathrm{B}, \mathrm{A}}=\mathrm{T}_{\mathrm{R}}=273 \mathrm{~K}$,

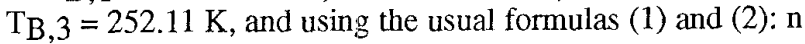
$=0.9196, \varepsilon=0.0002$

The beam filling factor is good, but we cannot measure any difference between the reflector and the lossless plate. This is indicated by the low emissivity value, and also evident from the bar diagram in Figure 5 .

Several experiments were carried out spread out over the period from August to December covering physical temperatures from $27^{\circ} \mathrm{C}$ to $-4^{\circ} \mathrm{C}$. In no case could any statistically significant difference be observed. This was also the case at $17 \mathrm{GHz}$. 


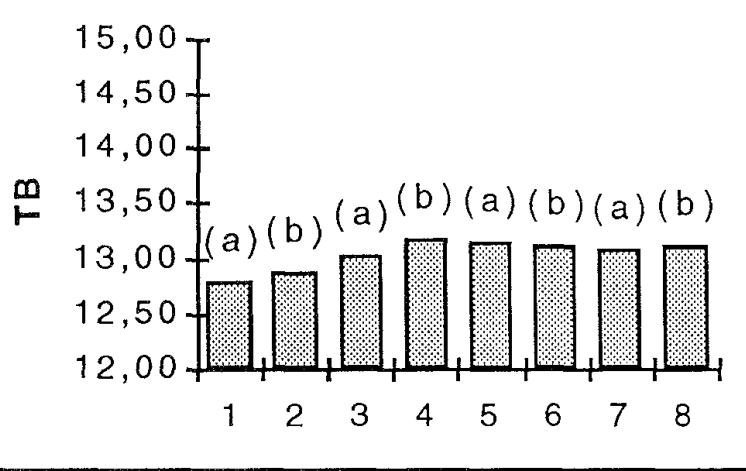

Figure 5: $34 \mathrm{GHz}$ measurement of the MIMR reflector

The $5 \mathrm{GHz}$ measurements are of special interest. The MIMR reflecter is manufactured in carbon fibre with a very thin metallization as reflecting surface. One could be nervous about the performance at the low frequency with its larger skin depth.

The $5 \mathrm{GHz}$ measurements start out with an example where the large standard gain horn is used, see Figure 6 . We find $n$ $=0.7589$, and $\varepsilon=-0.0043$. This measurement indicates that the MIMR reflector is better than the lossless plate which is of course nonsense. The beam filling factor is poor due to the large horn. In Figure 7 the situation is rectified by using the small horn. Here we find $n=0.8824$ and $\varepsilon=-0.0003$. Now we find the well known situation: provided satisfactory beam filling factor we cannot measure the difference between the MIMR reflector and the lossless plate.

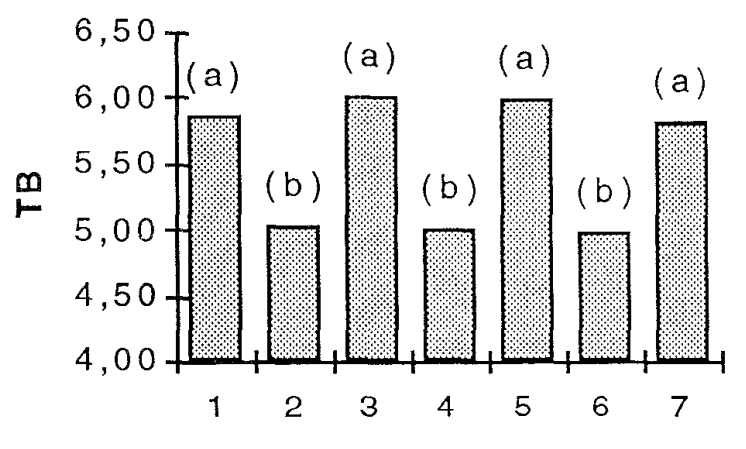

Figure 6: $5 \mathrm{GHz}$ measurement of the MIMR reflector, large horn.

The question of beam filling factor was examined by further experiments also at the higher frequencies by varying the distance between the horn and the reflector / lossless plate. As soon as the beam filling factor reaches close to $90 \%$ we get reasonable measurements, and things do not change even if the factor is further enlarged The explanation to this behaviour is sought in edge illumination problems: When the beam filling factor is inadequate for whatever reason (distance too large, horn too big) reflections / diffraction in the edges of the lossless plate contributes significantly to the received radiation. The bias by these contributions can only go in one direction since the nadir brightness temperature is the lowest possible and could thus explain our problems.

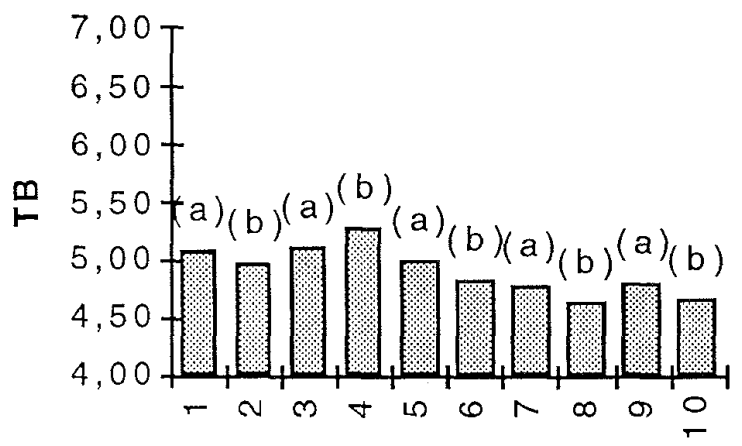

Figure 7: $5 \mathrm{GHz}$ measurements of the MIMT reflector, small horn

\section{DISCUSSIONS AND CONCLUSIONS}

- The experiments with different metal plates indicate that the present measurement setup cannot measure emissivities below 0.001 . It is for example obvious that we cannot measure the difference between aluminium and silver (the "lossless" plate), and the bucket can be regarded as lossless.

- The experiments with the MMR reflector show that in the frequency range $5-34 \mathrm{GHz}$ we cannot see any difference between the reflector and the lossless plate. It is believed that the reflector (which is manufactured in carbon fibre and coated with a thin layer of aluminium) concerning emissivity approaches that of an aluminium plate (emissivity in the range of $0.00024-0.00064$ ). This cannot, however, be proven by the present measurements due to the $\varepsilon=0.001$ limit stated above. But we can conclude that in the frequency range $5-34 \mathrm{GHz}$ the emissivity of the MIMR reflector is below 0.001 .

- Measurements have been carried out in the temperature range $+27^{\circ} \mathrm{C}$ to $-4^{\circ} \mathrm{C}$. No dependence on temperature is found.

- The sky brightness temperature reflected in a reflector having an emissivity of 0.001 and a physical temperature of $300 \mathrm{~K}$ is only modified by $0.3 \mathrm{~K}$. The somewhat higher brightness temperatures from Earth targets are modified correspondingly less (some $0.2 \mathrm{~K}$ worst case). This means that correction for MIMR main reflector losses are hardly warranted. $0.2 \mathrm{~K}$ must be included in the calibration budget.

- If better values for the emissivity of the MIMR reflector are needed the way forward is to adopt a slightly different measurement concept: a specimen of the reflector material having the shape and size of the lossless plate must be manufactured. The specimen and 
the lossless plate are again measured alternatively in the metal bucket. It is possible to make a mechanism so the specimen and the lossless plate can be interchanged in seconds - speed is a major factor considering instrument and atmosphere instabilities. At the same time geometry is consistent and edge effects will cancel. This way very accurate measurements are possible.

\section{ACKNOWLEDGMENTS}

The work described in the present paper was carried out under contract with the European Space Agency through Alenia Spazio. Mr. R. Bordi and Mr. P. Spera of Alenia have contributed to the work through fruitful discussions.

\section{REFERENCES}

Although not directly referred to during the work and in this paper the following references contain valuable information about the subject in question:

K. R. Carver; "Antenna and Radome Loss Measurements for MFMR and PIMS," Report, Physical Science Laboratory, New Mexico State University, 1975.

R. F. Harrison and H-J. C. Blume, "Determination of Electromagnetic Properties of Mesh Material Using Advanced Radiometer Techniques," NASA Langley Research Center. In NASA Conference Publication 2368: Large Space Antenna Systems Technology, 1984. 